\title{
POTENTIAL FUNCTIONS ON THE BOUNDARY OF THEIR REGIONS
}

\section{OF DEFINITION*}

BY

\section{O. D. KELLOGG}

In a memoir on PiCard's method of successive approximations, $\uparrow$ DinI points out the necessity of knowing that the potential functions used have first derivatives which are bounded even in the neighborhood of points of the boundary of their region of definition. These functions are determined by their boundary values, and for the case of the circle DINI finds the following condition on the boundary values $f(\vartheta)$ sufficient to insure limited derivatives for the potential function approaching them: $\left|f^{\prime}(\vartheta)\right|$ shall be finite and integrable, and the integral

$$
\int\left|\frac{f^{\prime}(\vartheta+t)-f^{\prime}(\vartheta-t)}{t}\right| d t
$$

shall be convergent when extended over an interval containing $t=0$ in its interior, for every value of $\vartheta . \ddagger$ Here $\vartheta$ is understood to be the angle subtended at the center by an arc of the circle between a fixed and a variable point. $\mathrm{He}$ then remarks that the theorem holds for other regions which can be conformally represented upon the circle by transformations which bring no singularities with them, clearly not intending this to be a complete answer to the problem, for the existence of the transformations mentioned is a special case of the general question of the behavior of the derivatives of a potential function defined by its boundary values. § Aside from its importance to the method of successive approximations, an investigation of the behavior of the derivatives of a potential

* Presented to the Socioty December 1, 1906. Received for publication July 11, 1907.

t Aota Mathematica, vol. 25 (1902), p. 185.

$\ddagger$ It seems worth noticing that the continuity of $f^{\prime}(\vartheta)$ is insufficient. For if $f^{\prime}(\vartheta)$ be defined in the neighborhood of $\vartheta=0$ as $\vartheta /|\vartheta| \sqrt{\log 1 / \mid \vartheta} \mid$ for $\vartheta+0$ and as $f^{\prime}(0)=0$ for $\vartheta=0$, and be elsewhere arranged to be continuous and periodic, the potential function approaching the boundary values $f(\vartheta)$ will have a derivative in the direction of the radius which becomes infinite in the neighborhood of the point $\vartheta=0$. On the other hand DinI's condition does not imply the continuity of $f^{\prime}(\vartheta)$.

8 The mapping is dependent apon the Green's function $\log 1 / \rho+v$, where $v$ is a potential function taking on the same boundary values as $-\log 1 / \rho$. But $\log 1 / \rho$ is analytic, so that the character of the derivatives of the mapping transformations is essentially that of the derivatives of $v$. 
function in the neighborhood of boundary points finds application in the theory of functions of a complex variable and in questions of mathematical physics, * notably such as make use of Green's functions.

The first treatment of this topic appears to be due to CAPELLI ; $\dagger$ his work for the general region depends upon a hypothesis regarding the behavior of a second derivative of the potential function. For the case of the circle he shows that the first and second derivatives of the potential function approaching the boundary values $f(\vartheta)$ are limited provided $f^{\prime}(\vartheta)$ exists and $\left[f^{\prime}(\vartheta+t)-f^{\prime}(\vartheta)\right] / t$ is limited. For the continuity of the first derivatives within and upon the boundary C. Neumann $\ddagger$ finds the conditions that the boundary curve have continuous curvature, and that the boundary values have a continuous first derivative and a second derivative which is continuous except at isolated points. Painleve § considers functions $X+i Y$ of a complex variable $x+i y$ in a region bounded by a curve $x=x(s), y=y(s), s$ being the length of are. If $x(s)$ and $y(s)$ have $p+2$ derivatives and if the function $X(s)$ which $X$ approaches on the boundary has an integrable $(p+1)$ th derivative, $X+i Y$ has limited derivatives of $p$ th and lesser orders. The conditions found by KoRN for the finiteness of the derivatives of potential functions of a three-dimensional region involve the existence of other functions taking on the required boundary values and satisfying certain continuity requirements on their derivatives of first and second orders. In all cases the results admit of the generalization contained in the following pages.

\section{§ 1. Definitions. Statement of hypotheses and main conclusions.}

In the present paper we shall deal with a simply connected closed region $R$ of the $x, y$-plane bounded by a curve $C$, the coördinates of whose points we shall think of as given by functions $x(s), y(s)$ of the length of arc $s$ measured from a fixed point. Points "within" $R$ means points of $R$ not belonging to $C$. A "potential function" of $R$ means a function $u$ of $x$ and $y$ which together with its first derivatives is continuous, and which has second derivatives satisfying the relation $\Delta u=\partial^{2} u / \partial x^{2}+\partial^{2} u / \partial y^{2}=0$ at all points within $R$. We shall be concerned with potential functions which approach boundary values $f(s)$ as $(x, y)$ approaches in any manner in $R$ a boundary point $[x(s), y(s)]$; the boundary

* See the important memoir of LIAPOUNOFF, Sur certaines questions qui se rattachent au problème de Dirichlet, Journal de Mathematiques, ser. 5, vol. 4 (1898), p. 241.

†Sopra l'integrale dell' equazione alle derivate potenziali di Laplace, Giornale di matematiohe di Battaglini, vol. 13 (1885), pp. 123-157.

$\ddagger$ Über die Methode des arithmetischen Mittels, Zweite Abhandlung, Leipziger Abhandlangen, vol. 14 (1888), pp. 562-726.

¿s Sur la theorie de la répresentation conforme, Compies Rendus, vol. 112 (1891), pp. 653-657.

II'ber die Lösungen des Dirichlet'schen Problems welche durch eine Combination der Methoden von Neumann und Schwarz gefunden verden, Mathematisobe Annalen, vol.53(1900), pp.593-608. 
values $f(s)$ we shall assume continuous. Such conditions are known to determine $u$ if it exists ; and $R$ is said to be the "region of definition" of $u$. We shall seek conditions upon $x(s), y(s)$ and upon $f(s)$ which suffice that the derivatives $\partial u / \partial x$ and $\partial u / \partial y$ approach finite values at each point $[x(s), y(s)]$ of the boundary, these values being independent of the manner of approach in $R$. From the conditions found it will also follow that these derivatives are bounded.

Previous investigations of this character have assumed a boundary curve with finite, if not continuous curvature at each point. Consideration of the conformal mapping problem and the Green's function, however, make it appear important to lessen the assumptions with respect to $C$, and, if necessary, to increase those upon $f(s)$. For the derivatives of the Green's function in the neighborhood of the boundary will evidently have the same continuity characteristics as those of of the additive potential function $v$ which takes on the same boundary values as $\log \rho$, that is, $f(s)=\frac{1}{2} \log \left[(\xi-x(s))^{2}+(\eta-y(s))^{2}\right]$. But these values have the same continuity characteristics as $x(s), y(s)$. We are thus led to seek conditions which bear, as nearly as possible, equally upon boundary curve and upon boundary values, a consideration which seems thus far to have escaped attention.

With this in mind one would be led to attempt to obtain DrNI's results for regions bounded by curves whose ${ }^{\circ} x(8)$ and $y(s)$ satisfy simply his conditions upon $f(\vartheta)$. That the results remain true is probable, but $I$ have not been able to attain quite this degree of generality. The results $I$ have succeeded in establishing may be stated as follows.

Let $R$ be a simply connected closed region of the $x, y$-plane bounded by a curve $C$ with parametric equations $x=x(s), y=y(s)$, where $s$ denotes the length of arc of $C$ measured from a fixed point, and let $f(s)$ be a function of the position of a point on $C$. We then have the following theorem.

(A) If three positive numbers $N, \alpha$ and $\delta$ independent of 8 and $\Delta s$ can be found such that

$$
\left|x^{\prime}(s+\Delta s)-x^{\prime}(s)\right|<N|\Delta s|^{a}, \quad\left|y^{\prime}(8+\Delta s)-y^{\prime}(s)\right|<N|\Delta s|^{a}
$$

as soon as $|\Delta s|<\delta$; ${ }^{*}$ and

* Conditions of this sort are fairly frequent in potential theory. If we omit the demand that $N, a$ and $\delta$ be independent of 8 , the functions $x^{\prime}(8), y^{\prime}(8)$ would be termed by KoRN (Lehrbuch der Potentialtheorie, vol. 2, p. vi) "regular," and the curve $C$ would be a "regular curve." As the word "regular" has been used in a different sense (see Encyklopädie der Mathematischen Wissenschaften, vol. 2, B. 1, p. 9, footnote) I should propose the more connotative expression, "algebraically continuous," suggested by the behavior of algebraic functions at branch-points where they are finite. The functions $x^{\prime}(s)$ and $y^{\prime}(s)$ of condition $(A)$ have algebraic continuity which is uniform, and the constant $\alpha$ we shall call the "index" of algebraio continuity. It is interesting to note that algebraic continuity in a closed interval does not imply uniform algebraic continuity. 
(B) if $f^{\prime}(8)$ is continuous and such that the integral

$$
\int_{0}^{\tau}\left|\frac{f^{\prime}(8+t)-f^{\prime}(8-t)}{t}\right| d t
$$

is convergent and vanishes with $\tau$ uniformly with respect to 8 ;

then there exists a uniquely determined potential function of $R, u(x, y)$, which approaches the boundary values $f(s)$; it has first derivatives which approach finite limits on the boundary and these limits form each a continuous function of 8 ; if $u(x, y)$ be defined at the boundary by the values it approaches, the (in general one-sided) derivatives $\partial u / \partial x$ and $\partial u / \partial y$ exist at the boundary and coincide with the limits of these derivatives formed for interior points, so that $\partial u / \partial x$ and $\partial u / \partial y$ are continuous in the closed region $R . \dagger$

The proof of this theorem depends upon the representation of a potential function as the potential of a double distribution on $C$. In a study of this question in a second paper by means of the method given by Fredholm, $\ddagger$ I shall show that $u(x, y)$ exists and is equal in the interior of $R$ to the potential $W(x, y)$ of a double distribution on $C$ with a moment $\phi(s)$ satisfying condition $(B)$. In this paper we shall limit ourselves to a study of the double distribution $W(x, y)$, and find that if its moment $\phi(s)$ satisfies condition $(B)$, the potential function $u(x, y)$ coinciding with $W(x, y)$ within $R$ and defined at the boundary so as to be continuous has the properties enunciated above. This will establish the theorem.

\section{§ 2. The normal derivatives of the potential of a double distribution.}

Let the potential of the double distribution be given in the form

$$
\begin{aligned}
& W(\xi, \eta)=\int_{0}^{l} \phi(t) \frac{\partial}{\partial t} \arctan \frac{y(t)-\eta}{x(t)-\xi} d t=2 \pi \phi(0)-\int_{0}^{l} \phi^{\prime}(t) \arctan \frac{y(t)-\eta}{x(t)-\xi} d t \\
& \text { or } \\
& W(\xi, \eta)=-\int_{0}^{l} \phi^{\prime}(t) \arctan \frac{y(t)-\eta}{x(t)-\xi} d t,
\end{aligned}
$$

* Two special cases may be mentioned in which condition $(B)$ may be lightened. First, if $R$ is the surface of a circle, the theorem holds if $f^{\prime}(s)$ is continnous, and if the integral

$$
\int_{0}^{r} \frac{f^{\prime}(s+t)-f^{\prime}(s-t)}{t} d t
$$

vanishes uniformly with $\tau$. Secondly, for the general region where $C$ sntisfies condition $(A)$ if we drop the demand for uniformity in $(B)$, we may still assert that the derivatives $\partial u / \partial x$ and $\partial u / \partial y$ approach finite limits as the point $(x, y)$ approaches a boundary point $[x(s), y(s)]$ along the normal through it, and these limits form each a continuous function of s. We cannot however argue either uniformity of the approach or that the limit is independent of the direction of the approach.

+ By "approach" in the above theorem is meant the general approach along any infinite set of points in $R$.

† Oefversigt af Kongl. Vetenskaps-Akademiens Förhandlingar, Stookholm, 1900. 
according as $(\xi, \eta)$ lies within or without $C ; l$ is the length of $C$. We first consider the derivative in the direction of a normal $\nu$ to $C$ through $(\xi, \eta)$. It is given by

where

$$
\frac{\partial W}{\partial \nu}=-\int_{0}^{l} \phi^{\prime}(t) \frac{\partial}{\partial \nu} \arctan \frac{y(t)-\eta}{x(t)-\xi} d t=\int_{0}^{l} \phi^{\prime}(t) \frac{\partial}{\partial \sigma} \log \frac{1}{\rho} d t,
$$

$$
\rho^{2}=[\xi-x(t)]^{2}+[\eta-y(t)]^{2}
$$

and where $\sigma$ denotes the direction perpendicular to $\nu$ and related to it as the positive $x$-axis to the positive $y$-axis. We shall prove in this section that as $(x, y)$ approaches $[x(8), y(s)]$ along the normal, $\partial W / \partial \nu$ approaches a finite limit; that this limit is the same whether the approach is from within or without $C$, and that the approach is in both cases uniform with respect to 8 . To do this we write

where

$$
\frac{\partial W}{\partial \nu}=J_{1}+J_{2}
$$

and

$$
J_{1}=\int_{0}^{\sigma-\tau} \phi^{\prime}(t) \frac{\partial}{\partial \sigma} \log \frac{1}{\rho} d t+\int_{s+\tau}^{l} \phi^{\prime}(t) \frac{\partial}{\partial \sigma} \log \frac{1}{\rho} d t
$$

$$
J_{2}=\int_{s-\tau}^{s+\tau} \phi^{\prime}(t) \frac{\partial}{\partial \sigma} \log \frac{1}{\rho} d t
$$

in which we impose upon $\tau$ a first restriction that a circle with center at $[x(s), y(s)]$ through the nearer of the two points $[x(s+\tau), y(s+\tau)]$ and $[x(s-\tau), y(s-\tau)]$ shall cut from $C$ only one arc, namely that passing through $[x(s), y(8)]$. As $C$ has a continuously turning tangent and no double points, one value for this upper limit for $\tau$ may be found which will serve for all values of 8 , as may be easily verified. Now for fixed $\tau$ the distance

$$
\rho(s, s+\tau)=\sqrt{[x(s+\tau)-x(s)]^{2}+[y(s+\tau)-y(s)]^{2}}
$$

has a positive minimum; call it $r$. Then within the circle

$$
[\xi-x(s)]^{2}+[\eta-y(s)]^{2}=(r / 2)^{2}
$$

$J_{1}$ is a continuous function* of $(\xi, \eta)$, a point on the normal to $C$ through $[x(s), y(s)]$, and the continuity is uniform with respect to $\xi, \eta$ and $s$. On the other hand it will be shown that $\tau$ may be taken so small that independently of $s$ the inequality $\left|J_{2}\right|<\epsilon / 4$ holds for any point $(\xi, \eta)$ of the normal, except $[x(s), y(s)], \epsilon$ being an arbitrary positive constant. Determining $\tau$ so as to meet both the above demands, and then $r$, we can, because of the continuity of

- In fact its first derivative is continuous and has a finite apper limit independent of 8 , being the second derivative of a potential within a region inoluded within a region containing no masses. 
$J_{1}$, determine $\kappa<r / 2$ such that for any two points $(\xi, \eta)$ and $\left(\xi^{\prime}, \eta^{\prime}\right)$ of the normal within the circle $[\xi-x(8)]^{2}+[\eta-y(8)]^{2}=\kappa^{2}$ we have

$$
\left|J_{1}\left(\xi^{\prime}, \eta^{\prime}\right)-J_{1}(\xi, \eta)\right|<\frac{\epsilon}{2}
$$

independently of 8 . Then also since

we have

$$
\left|J_{2}\left(\xi, \eta^{\prime}\right)-J_{2}(\xi, \eta)\right| \leqq\left|J_{2}\left(\xi, \eta^{\prime}\right)\right|+\left|J_{2}(\xi, \eta)\right|<\frac{\epsilon}{2},
$$

$$
\left|\frac{\partial}{\partial \nu} W\left(\xi, \eta^{\prime}\right)-\frac{\partial}{\partial \nu} W(\xi, \eta)\right|<\epsilon .
$$

Hence, $\partial W(\xi, \eta) / \partial \nu$ approaches a finite limit as $(\xi, \eta)$ approaches $[x(s), y(s)]$ along the normal to $C$ through this point, the approach of $\partial W(\xi, \eta) / \partial \nu$ being uniform with respect to 8 , and the limit being the same whether the approach of $(\xi, \eta)$ be from within or from without $C .^{*}$

To prove the stated property of $J_{2}$, let us take for axes the tangent and normal through $[x(s), y(8)]$, and for simplicity in writing let us measure 8 from this point as origin. Then the integral

$J_{2}=\int_{-\tau}^{+\tau} \phi^{\prime}(t) \frac{\partial}{\partial x} \log \frac{1}{\rho} d t=\int_{0}^{\tau}\left\{\phi^{\prime}(t) \frac{\partial}{\partial x} \log \frac{1}{\rho(t)}+\phi^{\prime}(-t) \frac{\partial}{\partial x} \log \frac{1}{\rho(-t)}\right\} d t$ may be written in the form $J_{21}+J_{22}$ where

and

$$
J_{21}=\int_{0}^{\tau}\left[\phi^{\prime}(t)-\phi^{\prime}(-t)\right] \frac{\partial}{\partial x} \log \frac{1}{\rho} d t
$$

$$
J_{22}=\int_{0}^{\tau} \phi^{\prime}(-t)\left\{\frac{\partial}{\partial x} \log \frac{1}{\rho(t)}+\frac{\partial}{\partial x} \log \frac{1}{\rho(-t)}\right\} d t .
$$

For the first of these two integrals we find an upper limit as follows:

$\left|J_{21}\right| \leqq \int_{0}^{r}\left|\phi^{\prime}(t)-\phi^{\prime}(-t)\right| \cdot\left|\frac{\partial}{\partial x} \log \frac{1}{\rho}\right| d t=\int_{0}^{r}\left|\phi^{\prime}(t)-\phi^{\prime}(-t)\right| \cdot\left|\frac{1}{\rho} \frac{\partial \rho}{\partial x}\right| d t$. In this we have

$$
\left|\frac{\partial \rho}{\partial x}\right|=|\cos (\rho, x)| \leqq 1 \text {, }
$$

and $\rho$ is not less than the absolute value of its projection $x(t)$. But the latter satisfies the condition

$$
|x(t)|=\frac{\left|\int_{0}^{x} \sqrt{2} d x\right|}{\sqrt{2}} \geqq \frac{\left|\int_{0}^{x} \sqrt{1+\left(\frac{d y}{d x}\right)^{2}} d x\right|}{\sqrt{2}}=\frac{|t|}{\sqrt{2}}
$$

\footnotetext{
* Compare the theorem of Liapousomp, loc. dit., p. 290.
} 
if we so restrict $\tau$ that

$$
\left|\frac{d y}{d x}\right|=\left|\frac{y^{\prime}(t)}{x^{\prime}(t)}\right|<1,
$$

and this is possible because $y^{\prime}(0)=0, x^{\prime}(0)=1$ and $x^{\prime}(t)$ and $y^{\prime}(t)$ are uniformly continuous. Moreover, the restriction can be made so as to hold equally for all values of $s$. Hence

$$
\left|J_{21}\right| \leqq \sqrt{2} \int_{0}^{r}\left|\frac{\phi^{\prime}(t)-\phi^{\prime}(t)}{t}\right| d t
$$

which by hypothesis $(B)$ can be made less than $\epsilon / 8$ by restricting $\tau$ sufficiently, the restriction holding uniformly with respect to 8 .

Turning to $J_{22}$, as $(\xi, \eta)$ is on the normal, we have $\xi=0$, and hence

so that

$$
J_{22}=\int_{0}^{r} \phi^{\prime}(-t)\left\{\frac{x(t)}{\rho^{2}(t)}+\frac{x(-t)}{\rho^{2}(-t)}\right\} d t,
$$

$$
\left|J_{22}\right| \leqq \max \left|\phi^{\prime}(t)\right| \int_{0}^{r}\left|\frac{x(t)}{\rho^{2}(t)}+\frac{x(-t)}{\rho^{2}(-t)}\right| d t .
$$

The integrand is a continuous function of $\tau$ and $\eta$ except at the point $(0,0)$. We shall show it to be always less in absolute value than a finite constant multiplied by $t^{a-1}$ where $\alpha$ is the index of algebraic continuity of condition $(A)$. Hence, as $\phi^{\prime}(t)$ is continuous, $\left|J_{22}\right|$ can be made less than $\epsilon / 8$ by restricting $\tau$, independently of the value of 8 , and hence $\left|J_{2}\right| \leqq\left|J_{21}\right|+\left|J_{22}\right|<\epsilon / 4$, as we were to shgw.

To prove the property stated for the integrand of $J_{22}$, note that because $x(0)=y(0)=y^{\prime}(0)=0$ and $x^{\prime}(0)=1$, we have by applying the law of the mean and using hypothesis $(A)$ :

$$
\begin{aligned}
x(t) & =x^{\prime}(\vartheta t) \cdot t=t\left(1+F \cdot t^{a}\right), \quad y(t)=F \cdot t^{1+a}, \\
x(-t) & =-t\left(1+F \cdot t^{a}\right), \\
\rho^{2}(t) & =t^{2}\left(1+F \cdot t^{a}\right)^{2}+\left(F \cdot t^{1+a}-\eta\right)^{2}, \\
\rho^{2}(-t) & =t^{2}\left(1+F \cdot t^{a}\right)^{2}+\left(F \cdot t^{1+a}+\eta\right)^{2},
\end{aligned}
$$

where each $F$ denotes a finite function and where we have supposed $t(\leqq \tau)<\delta$ in order that hypothesis $(A)$ might be in force. If now we write

and

$$
N(\eta, t)=x(t) \rho^{2}(-t)+x(-t) \rho^{2}(t)
$$

$$
D(\eta, t)=\rho^{2}(t) \rho^{2}(-t),
$$

the integrand in $\left|J_{22}\right|$ is $N(\eta, t) / D(\eta, t)$. Employing the equations (1) we find

$$
|N(\eta, t)|<G t^{1+a}\left(t^{2}+t|\eta|+\eta^{2}\right),
$$


where $G$ is a finite constant, and

$$
\begin{aligned}
D(\eta, t)=\rho^{2}(t) \rho^{2}(-t) & =\left(t^{2}+\eta^{2}+F \cdot t^{2+a}-F \cdot t^{1+a} \cdot \eta\right)\left(t^{2}+\eta^{2}+F \cdot t^{2+a}+F \cdot t^{1+a} \cdot \eta\right) \\
& >\left[t^{2}+\eta^{2}-G t^{a}(t+t|\eta|)\right]^{2} .
\end{aligned}
$$

If now $\tau$ be further so restricted that $G t^{a}<\frac{1}{2}$, that is, $\tau<1 /(2 G)^{1 / a}$, then

$$
\left|\frac{N(\eta, t)}{D(\eta, t)}\right|<\frac{4 G t^{a-1}\left(1+\frac{|\eta|}{t}+\frac{\eta^{2}}{t^{2}}\right)}{\left(1-\frac{|\eta|}{t}+\frac{2 \eta^{2}}{t^{2}}\right)^{2}} \leqq 4 G M t^{a-1},
$$

where $M$ is the maximum of the bounded function $\left(1+x+x^{2}\right) /\left(1-x+2 x^{2}\right)^{2}$. This gives us the desired inequality. Thus the result enunciated with respect to the normal derivatives is fully justified."

\section{§3. The tangential derivatives of the potential of a double distribution.}

A similar result may be obtained for the derivatives of $W(\xi, \eta)$ in a direction $\sigma$ perpendicular to the normal. Using the same coördinate system as before, we have

where

$$
\begin{aligned}
\frac{\partial}{\partial \sigma} W(\xi, \eta) & =\frac{\partial}{\partial \xi} W(\xi, \eta)=-\int_{0}^{l} \phi^{\prime}(t) \frac{\partial}{\partial \xi} \arctan \frac{\eta-y(t)}{\xi-y(t)} d t \\
& =\int_{0}^{l} \phi^{\prime}(t) \frac{\eta-y(t)}{\rho^{2}} d t=J_{1}+J_{2},
\end{aligned}
$$

$J_{1}=\int_{-l / 2}^{-\tau} \phi^{\prime}(t) \frac{\eta-y(t)}{\rho^{2}} d t+\int_{\tau}^{2 / 2} \phi^{\prime}(t) \frac{\eta-y(t)}{\rho^{2}} d t$ and $J_{2}=\int_{-\tau}^{\tau} \phi^{\prime}(t) \frac{\eta-y(t)}{\rho^{2}} d t$.

Again $J_{1}$ is continuous for fixed $\tau$, and uniformly so in $\xi, \eta$ and 8 . If $J_{2}=J_{21}+J_{22}$, where

and

$$
J_{21}=\int_{0}^{\tau}\left[\phi^{\prime}(t)-\phi^{\prime}(-t)\right] \frac{\eta-y(t)}{\rho^{2}} d t
$$

$$
J_{22}=\int_{0}^{r} \phi^{\prime}(-t)\left\{\frac{\eta-y(t)}{\rho^{2}(t)}+\frac{\eta-y(-t)}{\rho^{2}(-t)}\right\} d t,
$$

then $J_{21}$ again vanishes with $\tau$ independently of $\eta$ and $s$. However, $J_{22}$ de-

* To obtain an expreseion for the limit of the normal derivatives of $W(\xi, \eta)$, which we denote by $W_{n}(s)$, a slight extension of the above work leads us to the result

$$
W_{n}(s)=\int_{0}^{t} \phi^{\prime}(t) \frac{\partial}{\partial s} \log \frac{1}{\bar{p}} d t
$$

where $\bar{p}^{2}=[x(t)-x(s)]^{2}+[y(t)-y(s)]^{2}$ and where the Cauchy prinoipal value of this integral is to be taken. This may be proven continuous by a direct examination. We shall have another prool of the continuity of $W_{n}(s)$ later. 
mands a different treatment, for it does not vanish with $\tau$. We put $J_{22}=J_{221}+J_{222}$, where

and

$$
J_{221}=2 \int_{0}^{r} \phi^{\prime}(-t) \frac{\eta}{\eta^{2}+t^{2}} d t
$$

$$
J_{222}=\int_{-\tau}^{+\tau} \phi^{\prime}(-t)\left\{\frac{\eta-y(t)}{\rho^{2}(t)}-\frac{\eta}{\eta^{2}+t^{2}}\right\} d t .
$$

Here $J_{221}=2 \phi^{\prime}(-\bar{t}) \arctan \tau / \eta$ where $0 \leqq \bar{t} \leqq \tau$ and $0 \leqq \arctan \tau / \eta \leqq \pi / 2$. To fix ideas, let us suppose we are approaching the boundary from within. It is legitimate to restrict $\eta$ to be less than $\tau^{2}$. Then

$$
\arctan \frac{1}{\sqrt{\eta}}<\arctan \frac{\tau}{\eta}<\frac{\pi}{2}
$$

thus by restricting first $\tau$ and then $\eta$ we can make $\phi^{\prime}(-t)$ as close to $\phi^{\prime}(0)$ and $\operatorname{arc} \tan \tau / \eta$ as close to $\pi / 2$ as we please, and accordingly we may make

$$
\left|J_{221}-\pi \phi^{\prime}(0)\right|<\frac{\epsilon}{12} .
$$

Hence for any two points $(0, \eta)$ and $\left(0, \eta^{\prime}\right)$ so restricted we have

$$
\left|J_{221}\left(0, \eta^{\prime}\right)-J_{222}(0, \eta)\right|<\epsilon / 6 \text {. }
$$

For $J_{222}$ we find by use of equations (1),

$$
\begin{aligned}
& \left|J_{222}\right| \leqq \max \left|\phi^{\prime}(t)\right| \cdot \int_{-\tau}^{+\tau}\left|\frac{y(t)\left\{\eta^{2}-\eta y(t)-t^{2}\right\}+\eta\left\{t^{2}-x^{2}(t)\right\}}{\rho^{2}(t) \cdot\left\{\eta^{2}+t^{2}\right\}}\right| d t \\
& <\max \left|\phi^{\prime}(t)\right| \cdot G \int_{-\tau}^{+\tau}|t|^{\alpha-1} \frac{\frac{\eta^{2}}{t^{2}}+\frac{\eta}{|t|}+1}{\frac{\eta^{2}}{t^{2}}+\frac{1}{2}} d t \leqq \max \left|\phi^{\prime}(t)\right| \frac{2 G M \tau^{a}}{\alpha},
\end{aligned}
$$

where $M$ is the maximum of the bounded function $\left(x^{2}+|x|+1\right) /\left(x^{2}+\frac{1}{2}\right)$. Thus $\tau$ can be so restricted that independently of $\eta$ we have $\left|J_{222}(0, \eta)\right|<\epsilon / 12$, and hence

$$
\left|J_{222}\left(0, \eta^{\prime}\right)-J_{222}(0, \eta)\right|<\frac{\epsilon}{6} .
$$

We now fix $\tau$ so that $\left|J_{21}\right|<\epsilon / 12$, and therefore $\left|J_{21}\left(0, \eta^{\prime}\right)-J_{21}(0, \eta)\right|<\epsilon / 6$; also so that (3), and with the proper additional restriction upon $\eta$, so that (2) shall hold; then from these three inequalities we find $\left|J_{2}\left(0, \eta^{\prime}\right)-J_{2}(0, \eta)\right|<\epsilon / 2$ for all values of $\eta$ and $\eta^{\prime}$ between 0 and an ascertainable positive number $\kappa$. Then as $J_{1}(0, \eta)$ is continuous we may, if necessary, further restrict $\kappa$ so as 
to obtain the inequality $\left|J_{1}\left(0, \eta^{\prime}\right)-J_{1}(0, \eta)\right|<\epsilon / 2$, whence follows

$$
\left|\frac{\partial}{\partial \xi} W\left(0, \eta^{\prime}\right)-\frac{\partial}{\partial \xi} W(0, \eta)\right|<\epsilon .
$$

The same inequality will be found for $-\kappa<\eta, \eta^{\prime}<0$.

Hence, $\partial W(\xi, \eta) / \partial \sigma$ approaches a finite limit as $(\xi, \eta)$ approaches $[x(s), y(s)]$ along the normal to $C$ from within; it also approaches a finite limit from without; each approach is uniform with respect to s."

\section{§4. Generalizations.}

The desired generalizations are immediate. First, denoting by $\mu$ any fixed direction, since

$$
\frac{\partial}{\partial \mu} W(\xi, \eta)=\frac{\partial W}{\partial \sigma} \cos (\sigma, \mu)+\frac{\partial W}{\partial \nu} \cos (\nu, \mu),
$$

we have the result;

$\partial W(\xi, \eta) / \partial \mu$ approaches a finite limit as $(\xi, \eta)$ approaches $[x(8), y(s)]$ along the normal to $C$ from within; it also approaches a finite limit from without; both approaches are uniform with respect to 8 .

Secondly, the limits form continuous functions of 8 . Let us denote the limit of $\partial W(\xi, \eta) / \partial \mu$ by $W_{m}(8)$, and to fix ideas let us consider the approach from within $C$. Suppose $W_{m}(8)$ were not continuous at a point 8 . Then we could find a positive quantity $\epsilon$ such that

$$
\left|W_{m}(8+\Delta s)-W_{m}(s)\right|>2 e,
$$

for certain values of $|\Delta s|$ as small as we please. But the approach of $\partial W / \partial \mu$ along the normal to its limit being uniform we can so determine $\alpha$ that as soon as the distance of $(\xi, \eta)$ from the foot of the normal is less than $\alpha$, the difference between $\partial W / \partial \mu$ and its limit is less than $\epsilon / 2$. That is, if

then

$$
\begin{array}{ll}
\xi=x(8)-\lambda y^{\prime}(8), & \xi^{\prime}=x(8+\Delta s)-\lambda y^{\prime}(s+\Delta s), \\
\eta=y(8)+\lambda x^{\prime}(8), & \eta^{\prime}=y(8+\Delta s)+\lambda x^{\prime}(8+\Delta s),
\end{array}
$$

(6) $\left|\frac{\partial}{\partial \mu} W(\xi, \eta)-W_{m}(8)\right|\left|<\frac{\epsilon}{2}, \quad\right| \frac{\partial}{\partial \mu} W\left(\xi^{\prime}, \eta^{\prime}\right)-W_{m}(8+\Delta s) \mid<\frac{\epsilon}{2}$,

* The limit from within will be found to be

and from without,

$$
\pi \phi^{\prime}(s)-\cdot \int_{0}^{t} \phi^{\prime}(t) \frac{\partial}{\partial n} \log \frac{1}{\bar{\rho}} d t ;
$$

$$
-\pi \phi^{\prime}(s)-\int_{0}^{t} \phi^{\prime}(t) \frac{\partial}{\partial n} \log \frac{1}{\bar{\rho}} d t \text {. }
$$

The integrals here are absolutely convergent and are continuous in 8. Cf. note, p. 46. 
whence from (4), (5) and (6)

$$
\left|\frac{\partial}{\partial \mu} W\left(\xi^{\prime}, \eta^{\prime}\right)-\frac{\partial}{\partial \mu} W(\xi, \eta)\right|>\epsilon
$$

for values of $|\Delta s|$ as small as we please. But from (5) it is evident that by decreasing $\Delta_{s}$ we can bring $\left(\xi^{\prime}, \eta^{\prime}\right)$ as near to $(\xi, \eta)$ as we please. The inequality (7) therefore means that the derivative $\partial W / \partial \mu$ of a potential is discontinuous at a point outside the attracting masses, which is known to be untrue. Hence, the limit $W_{m}(s)$ of the derivative $\partial W / \partial \mu$ in any fixed direction as $(\xi, \eta)$ approaches $[x(s), y(s)]$ along the normal forms a uniformly continuous function of 8 ; this is true for the limit from without as well as for the limit from within.

Thirdly, we may remove the restriction that the approach be along the normal. That is, we shall show that given any positive $\epsilon$ we can find a positive $r$ such that for all points $\left(\xi^{\prime}, \eta^{\prime}\right)$ of $R$ within the circle

$$
[\xi-x(8)]^{2}+[\eta-y(8)]^{2}=r^{2},
$$

we have the inequality

$$
\left|\frac{\partial}{\partial \mu} W\left(\xi^{\prime}, \eta^{\prime}\right)-W_{m}(s)\right|<\epsilon .
$$

To do this choose $\alpha$ as above, then choose $\beta<\alpha / 2$.so that

$$
\left|W_{m}(s+\Delta s)-W_{m}(s)\right|<\frac{\epsilon}{2}
$$

as soon as $|\Delta s|<\beta$. Then a positive quantity $r$ can be found such that for any point $\left(\xi, \eta^{\prime}\right)$ within the circle $[\xi-x(8)]^{2}+[\eta-y(8)]^{2}=r^{2}$ the second pair of equations (5) admit a solution $(\lambda, \Delta s)$ satisfying the inequalities $|\lambda|<\alpha$, $|\Delta s|<\beta$, and hence the second inequality (6), and also (8), are in force. Bu $r$ from them we have

$$
\left|\frac{\partial}{\partial \mu} W\left(\xi, \eta^{\prime}\right)-W_{m}(s)\right|<\epsilon .
$$

Hence $\partial W(\xi, \eta) / \partial \mu$ approaches its limit $W_{m}(s)$ for any manner of approach in $R$; a similar result holds for the region $R^{\prime}$ without $C$.

§5. The derivatives on the boundary of a potential function coinciding at interior points of $\boldsymbol{R}$ with the potential of a double distribution.

It is well known that $W(\xi, \eta)$ is in general discontinuous at points of $C$, so that there can ordinarily be no question of derivatives at points of $C$. However, if $u(\xi, \eta)$ is a potential function coinciding with $W(\xi, \eta)$ at all interior

\footnotetext{
* This simple theorem of analysis situs admits an easy proof. The number $r$ can be chosen independent of 8 .
}

Trans. Am. Math. Soc. 4 
points of $R$, it may be made continuous at all points of $C$ by defining it by its limiting values, $f(s)$; ; it will then have finite (in general one-sided) derivatives at each point of $C$, which coincide with the limits of the derivatives formed in the same direction at interior points of $R$. To show this, let us take the point $C$ in question as origin, and a positive $x$-axis pointing into $R$ in the given direction. Then $u(\xi, 0)$ is a continuous function of $\xi$ in a closed ascertainable interval beginning with $(0,0)$; it has a continuous derivative $\partial u(\xi, 0) / \partial \xi=\partial W(\xi, 0) / \partial \xi$ in the interior of the interval, and hence we may apply the law of the mean :

$$
\frac{\partial}{\partial \xi} u(0,0)=\lim _{h=0} \frac{u(h, 0)-u(0,0)}{h}=\lim _{h=0} \frac{\partial}{\partial h} u(\vartheta h, 0) \quad(0<\vartheta<1) .
$$

But because of the continuity of $\partial u(h, 0) / \partial h=\partial W(h, 0) / \partial h$ and because of the existence of its limit, $\partial u(0,0) / \partial \xi$ exists and equals $W_{h}(s)$.

Hence the potential function $u(\xi, \eta)$ has continuous first derivatives in the closed region $R$. $\dagger$

Columbia, Missouri, June, 1906.

* See p. 42.

T The same result holds for the potential function $u^{\prime}(\xi, \eta)$ of the region $R^{\prime}$ consisting of all points outside of and upon $C$. To the definition of $u^{\prime}(\xi, \eta)$ must then be added certain wellknown restrictions regarding its behavior at infinity. 